\title{
BERICHT \\ DES HISTORISCHEN INSTITUTS \\ BEIM ÖSTERREICHISCHEN KULTURFORUM IN ROM FÜR DAS STUDIENJAHR 2002/2003
}

I. Kuratorium der Österreichischen Akademie der Wissenschaften

Vgl. Almanach der Österreichischen Akademie der Wissenschaften 153 (2002/2003), 379 .

\section{Personalstand}

Vgl. Almanach der Österreichischen Akademie der Wissenschaften 153 (2002/2003), 380 .

\section{STIPENDiaten*}

819. (456.) Mag. Peter Erhart, I, G (Monastische Schriftkultur in Mittel- und Süditalien in langobardischer Zeit). Dreimonatiges Stipendium

820. (457.) Mag. Ingrid Gössinger, II, G (Die Europäische Verteidigungsgemeinschaft aus sowjetischer Sicht oder Die sovetischen Auseinandersetzungen um die Westintegration der Bundesrepublik Deutschland und ihre Bewaffnung im Rahmen der EVG. 1950-1954). Dreimonatiges Stipendium.

821. (458.) Mag. Regina Hanslmayr, I, A (Hermen aus Kleinasien). Viermonatiges Stipendium.

* Die Nummern des Verzeichnisses schließen an jene der in den RHM 45 (2003), 13f. enthaltenen Übersicht der wissenschaftlichen Stipendiaten an. - Verwendete Abkürzungen: A = Archäologie; $\mathrm{G}=$ Geschichte; $\mathrm{MG}=$ Musikgeschichte. - Die römischen Zahlen nach den einzelnen Namen bedeuten die Zahl der den Stipendiaten bisher zuerkannten Stipendien am Historischen Institut in Rom. 
822. (459.) Mag. Stefan Ikarus Kaiser, I, MG (Die Musiktheorie des Aristoxenos von Tarent. Literarische Zeugnisse zu Lehre und Person des Aristoxenos ). Viermonatiges Stipendium.

823. (460.) Mag. Thomas Kuster, I, G (Edition des italienischen Reisetagebuches Kaiser Franz' I. von Österreich 1819). Sechsmonatiges Stipendium.

824. (461.) Mag. Corinna Lorenzi, I, G (Diplomatische Beziehungen zwischen Österreich und dem Königreich Piemont-Sardinien zwischen 1815 und 1848). Sechsmonatiges Stipendium.

825. (462.) Mag. Georg Plattner, I, A (Transfer von Architekturkonzepten und Ornamentformen zwischen Kleinasien und Rom in der Kaiserzeit). Sechsmonatiges Stipendium.

826. (463.) Mag. Jane Regenfelder, I, G (Die politischen Dimensionen des „Bolzano-Prozesses”. Der „Weise von Prag”: Revolutionär oder Märtyrer der Metternichschen Reaktion?). Einmonatiges Stipendium.

827. (464.) Mag. Elina Talakovics, I, G (Das Regnum Italiae in salischer Zeit - Markgrafen und Bischöfe als politische Kräfte in den Regionen Toskana, Umbrien und Latium). Dreimonatiges Stipendium.

\section{Bibliothek und Archiv}

Die Bibliothek des Instituts ist im Berichtszeitraum um 1003 Bände vermehrt worden, somit auf über 54.000 Bände angewachsen, was mit den Sondersammlungen einen Gesamtbestand von ca. 82.000 Bänden ergibt. Die retrospektive Katalogisierung des Gesamtbestandes (inklusive Partituren) ist nun zu 90\% abgeschlossen und damit auch im Internet abrufbar. Derzeit wird der Zeitschriften-Fortsetzungskatalog von der Karteiform auf das EDV-Eintragungs- und Kontrollsystem des Bibliotheksnetzes URBS übertragen.

\section{VeröfFentlichungen}

1. Römische Historische Mitteilungen, 45. Band. Wien 2003. Verlag der Österreichischen Akademie der Wissenschaften. 445 S., 69 Abb.

2. Innocenzo III. Urbs et Orbis. Atti del Congresso Internazionale (Roma, 9-15 settembre 1998), hrsg. von Andrea Sommerlechner (Istituto Storico Italiano per il Medioevo. Nuovi Studi Storici $55=$ Miscellanea 
della Società Romana di Storia Patria XLIV). Rom 2003. 2 Bde., 1373 S., 91 Abb.

\section{Vorträge und Veranstaltungen}

1. Präsentation der Neuauflage des Buches „Austria e Italia. Storia a due voci" von Silvio Furlani und Adam Wandruszka, mit Beiträgen von Brigitte Mazohl-Waldnig (Universität Innsbruck), Maddalena Guiotтo (Centro per gli studi storici italo-germanici, Trient) und Stefan Malfér (Österreichisches Ost- und Südosteuropainstitut, Wien) (13. Februar 2003).

2. Internationale Tagung „Chiesa, politica e vita culturale negli ultimi decenni della Monarchia Austro-Ungarica“, in Zusammenarbeit mit der Accademia d'Ungheria in Roma, mit Vorträgen von Lászlo Németh (Istituto Pontificio Ungherese), Fidel González-Fernández (Pontificie Università Gregoriana e Urbaniana), Moritz Csáky (Universität Graz/ ÖAW Wien), Emilia Hrabovec (Universität Wien), Éva Somogy (Historisches Institut der Ungarischen Akademie der Wissenschaften, Budapest) und Lászlo Csorba (Accademia d'Ungheria in Roma) (27. März 2003).

3. Internationale Tagung ,Sir Ernst H. Gombrich. Storico dell'arte, Vienna 1909-Londra 2001“, mit Vorträgen von Paolo FABBRI (Università di Bologna), Michele Di Monte (Università Ca’ Foscari, Venezia), Maurizio Ferraris (Università di Torino), Massimo Carboni (Università della Tuscia, Viterbo), Augusto Gentili (Università Ca' Foscari, Venedig), Claudia Cieri Vıa (Università degli Studi di Roma „La Sapienza“), Luca Bortolotтr (Università Ca’ Foscari, Venezia), Gottfried Вӧнм (Universität Basel), Maria Giuseppina Di Monte Nisini (Galleria Nazionale d'Arte Moderna, Roma), Karl Clausberg (Universität Lüneburg), Nigel SPIvey (University of Cambridge), Michael Podro (University of Essex), Artur Rosenauer (Universität Wien) und Wolfgang Georg Fischer (Wien) in Zusammenarbeit mit der Bibliotheca Hertziana (Max-Planck-Institut), The British School at Rome, Galleria Nazionale d'Arte Moderna, Rom; Università Ca' Foscari, Venedig; Comune di Roma (31. März/1. April 2003).

4. Studientag „Mezzo secolo di studi su Innocenzo III. Bilancio delle ricerche e prospettive per la storia del papato nel Duecento", mit Beiträgen von P. Sergio Pagano (Archivio Segreto Vaticano, Vatikanstadt), Othmar Hageneder (Institut für Österreichische Geschichtsforschung, Wien), Martin Bertram (Deutsches Historisches Institut, 
Rom), Andrea Giuseppe Piazza (Istituto Storico Italiano per il Medioevo, Rom) in Zusammenarbeit mit dem Istituto Storico Italiano per il Medioevo (28. April 2003).

5. Vortrag von Mons. Walter Branduúller: „Marco d'Aviano (16311699) zwischen Spiritualität und Politik“, in Zusammenarbeit mit der Österreichischen Botschaft beim Heiligen Stuhl (29. April 2003).

6. Vortrag von Walter PoHL (Forschungsstelle für Geschichte des Mittelalters an der Österreichischen Akademie der Wissenschaften, Wien): „Alla ricerca delle origini etniche. Problemi d'identità tra antichità e medioevo" (27. Mai 2003).

\section{Vorträge von Angehörigen des Instituts}

1. Andreas GotTsmann: Vortrag „I rapporti politici e istituzionali tra il Veneto e l'area centroeuropea nell'Ottocento" im Rahmen der Tagung „Vie di Comunicazione in Mitteleuropa" (Castelbrando/ Treviso, 26. September 2002).

2. Andreas Gottsmann: Vortrag „Sprache und Schule im Küstenland“ im Rahmen der Tagung zu Sprache und Schule in der Habsburgermonarchie (Prag, Tschechische Akademie der Wissenschaften, Historisches Institut, 7. November 2002).

3. Karl Rudolf: Festvortrag „Ferdinand I. (1503-1564)“ im Rahmen der Veranstaltungen der Stadt Alcalá de Henares aus Anlass der 500. Wiederkehr des Geburtstages von Kaiser Ferdinand I. (Alcalá de Henares, 10. März 2003).

4. Richard Bösel: Vortrag „L'Architettura degli ordini religiosi della controriforma a Roma“" (Rom, Università degli Studi di Roma 2, Facoltà di Ingegneria, 15. April 2003).

5. Richard Bösel: Vortrag „Borromini - Tagliacantone. A proposito della poetica di un architetto angoloso" (Mendrisio/Ticino, Accademia di Architettura dell'Università della Svizzera Italiana, 21. Mai 2003).

6. Richard Bösel: Vortrag „L'architettura gesuitica siciliana nel contesto internazionale“ im Rahmen des Studientags „Architettura e urbanistica della Compagnia di Gesù in Sicilia“ (Trapani, Assessorato Regionale dei Beni Culturali ed Ambientali e della Pubblica Istruzione, Centro Studi G. B. Amico, 23. Mai 2003).

7. Richard Bösel: Vortrag ,Tipologia gesuitica, una valida categoria critica?" und didaktische Mitwirkung am 3. Forschungs-Seminar im Rahmen des Dottorato di Storia dell'architettura e dell'urbanistica 
„Architettura del Cinque-Seicento" (Venedig, Istituto Universitario di Architettura di Venezia, 3.-5. Juni 2003).

8. Andrea Sommer-Mathis: Vortrag „Immagine e Musica. Il mondo della festa alla corte viennesa di Leopoldo I e di Margarita d'Austria" im Rahmen des Vortragszyklus „L'Arte Barocca e l'ideale classico. Aspetti dell'arte cortigiana della seconda metà del Seicento" (Rom, Accademia Spagnola, 5. Juni 2003).

9. Rainer Murauer: Vortrag „Hanc poenam ecclesia non imponit“: Canonistic Doctrine on the Death Penalty, 1140-1234" im Rahmen des International Medieval Congress (Leeds, 15. Juli 2003).

10. Richard Bösel: Vortrag „Progetti inediti per il santuario ignaziano nel Gesù di Roma“ im Rahmen des Convegno internazionale „Per la storia dell'arte in Italia e in Europa. Studi in memoria di Luisa Mortari“ (Università degli Studi di Bari, 18. September 2003). 
